\title{
Mechanical Reinforcement in Polymers/graphite Composites Prepared in Bulk and Membrane Forms
}

\author{
M. Amin', G. M. Nasr ${ }^{a}$, G. H. Ramzy ${ }^{a}$, and E. Omar ${ }^{b *}$. \\ ${ }^{a}$ Physics department, Faculty of science, Cairo University, Giza, Egypt. \\ ${ }^{b}$ Egyptian Petroleum Research Institute (EPRI), Cairo, Egypt \\ emo.star2006@gmail.com
}

\begin{abstract}
Two different Composite samples of NR and IIR were prepared with graphite as reinforcing filler in bulk and membrane forms. Tensile tests were performed at room temperature to explore the rule of graphite on the mechanical reinforcement of these composites. For bulk form, tensile modulus was found to increase with filler loading which could be explained using the percolation theory. Different models were applied to account for the experimental data. For NR in the membrane form, the percolation threshold is lower than that in the bulk form while the tensile modulus has increased to about 18 times than its value in the bulk state for NR.
\end{abstract}

\section{Introduction}

Elastomeric compounds are a class of materials widely used not only in general products, but also in specialized fields such as aerospace, biomedical, micro-electromechanical systems and shape memory polymers [1-3]. In many of these applications, high strength, superior electrical and thermal conductivities, and improved tribological and barrier properties are required. Various types of filler have been incorporated into rubber matrices in order to provide reinforcement or functional properties. However, most of those filled composites do not combine enhanced mechanical properties with superior multifunctional performance. Thus, graphite may be an ideal nanofiller to impart these mechanical [4,5] properties to rubber materials, provided that fine dispersion of the filler and strong interfacial interaction between filler and rubber are achieved.

He et al [6], prepared Poly (vinylidene fluoride) (PVDF)/graphite composites. These composites exhibited much higher storage modulus 
compared to those of pure PVDF, especially when the graphite loading amount was $10 \mathrm{wt} \%$. Song et al [7], prepared the graphite/carbon black/SBR composites. Tensile properties of the composites indicated higher modulus, tensile strength and elongation in comparison with composites of pristine graphite, carbon black. Also, the composites were found to be in improving tendency with thermal properties and fatigue properties. Sarikanat et al [8], fabricated high density polyethylene (HDPE)/graphite nanocomposite and an increase in tensile strength and modulus of elasticity were observed with increasing graphite powder content from 0 to $6 \%$. However, for a further increase in the graphite content; tensile strength decreases while modulus of elasticity continued to increase in the composite. More recently, Wang et al [9], prepared acrylonitrile-butadiene rubber (NBR)/expanded graphite (EG)/carbon black (CB) by two different methods, They found the modulus of the NBR/EG/CB composites is higher than that of NBR/CB composite because of the higher dispersion of graphite sheets lead to a significant enhancement on tensile modulus (at 100\% elongation) and hardness. Raza et al [10], prapared graphite nanoplatelets (GNPs) / silicone polymer using a three-roll mill process. The composites moduli increased by 1.5 times up to $20 \mathrm{wt} . \%$ loading but the strength and strain to failure decreased.

Obviously from above mention efforts, graphite is one of the most important inorganic fillers which plays a major role in today's science and technology and are required to advance with better properties to meet new requirements or to replace existing materials.

In this research we continue to explore the role of preparation method and the final form of the product on mechanical properties of the prepared composites as a apart of a complete study of its role on the physical properties in general [11].

\section{Experimental work:}

\section{Materials used in this Work}

Natural rubber (NR) and butyl rubber (IIR) which were supplied by TRENCO, Alexandria, Egypt and Fine powder extra pure graphite $(50 \mu \mathrm{m})$ was supplied by Merck, Germany were used in this study. Graphite properties are: Solubility $\left(20{ }^{\circ} \mathrm{C}\right)$ insoluble; Molar mass $12.01 \mathrm{~g} / \mathrm{mol}$; Density $2.2 \mathrm{~g} / \mathrm{cm}^{3}\left(20{ }^{\circ} \mathrm{C}\right)$; Bulk density $280 \mathrm{~kg} / \mathrm{m}^{3} ; \mathrm{pH}$ value $5-6\left(50 \mathrm{~g} / \mathrm{l}, \mathrm{H}_{2} \mathrm{O}, 20^{\circ} \mathrm{C}\right)$. The calculation of the true graphite volume fraction was performed through the following relationship

$$
\varphi_{\text {true graphite }}=\frac{w_{\text {true graphite }} / \rho_{\text {true graphite }}}{\sum_{i} w_{i} / \rho_{i}}
$$

Where $w_{i}$ and $\rho_{i}$ are the weight fraction and the density of the $i^{\text {th }}$ phase, respectively. 
2. Sample preparation and Measurements

\subsection{Bulk Forms}

All samples were prepared according to the following method with the compositions shown in Tables (1) \& (2). Ingredients of the rubber composites were mixed on a 2-roll mill of $170 \mathrm{~mm}$ diameter, working distance $300-\mathrm{mm}$, speed of slow roll being $24 \mathrm{rpm}$ and gear ratio 1.4. The compounded rubber was divided into two groups; the first group was left for 24 hours before vulcanization. The vulcanization process was performed at $153 \pm 2{ }^{\circ} \mathrm{C}$ under a pressure of 150 bar for 15 minutes. To ensure reproducibility of the results, the samples were aged at $70^{\circ} \mathrm{C}$ for 10 days. By this way the bulky samples were prepared with average thickness $0.3 \mathrm{~cm}$.

\subsection{Membrane Form}

This group was prepared by dissolving in methylbenzene to obtain a highly concentrated solution. Subsequently, each gelatinous solution was shaped into membrane in a form of circle $(7 \mathrm{~cm}$ in diameter) by means of a stainless steel dish. After slow drying, a smooth and uniform thin composite membrane was obtained about $0.7 \mathrm{~mm}$ in thickness. Then all membrane samples were vulcanized under pressure of $294 \mathrm{bar}$, at temperature of $153^{\circ} \mathrm{C}$, and time of 30 minutes, the final membranes have average thickness $0.4 \mathrm{~mm}$ for NR samples and $0.2 \mathrm{~mm}$ for IIR samples. Finally they were aged at $70{ }^{\circ} \mathrm{C}$ for 10 days to insure reproducibility of results. All measurement was registered using a homemade tensile-testing machine connected with a force gauge and controlled strain rate.

\section{Results and discussion \\ Bulk Samples}

Tensile tests performed at room temperature and at a crosshead speed of 100 $\mathrm{mm} / \mathrm{min}$ on the material of natural (NR) and butyl rubber (IIR) are reported in Figures (1) and (2) respectively. The results reveal a progressive increase of both material stiffness and strength by increasing the amount of graphite. Also, appreciable decrease in elongation at break is also found for samples with graphite loading higher than $40 \mathrm{phr}$ graphite content.

On calculating the initial modulus of both filled NR and IIR matrices as the slope of the low strain region of the nominal stress- nominal strain curves, we find that the initial modulus has increased from $1 \mathrm{MPa}$ to $3.7 \mathrm{MPa}$ for NR and form $0.065 \mathrm{MPa}$ to $3 \mathrm{MPa}$ for IIR on increasing the graphite loading from $0 \mathrm{phr}$ to $100 \mathrm{phr}$ (corresponding to filler volume fraction 0 and 0.4 respectively). In other words, the modulus has increase by $370 \%$ and $460 \%$ for NR and IIR respectively for such filler loadings. However, as observed from Figures (3), and (4), the increase is linear with low filler loading, and the behavior deviates from linearity for loadings above a certain critical value known as the 
percolation threshold. However, we shall try to explain such behavior on the basis of the percolation theory.

Rigid fillers typically increase the stiffness of an elastomeric material. A theory for the stiffening of elastomer by rigid fillers is based on the Einstein's theory $[12,13]$ for the increase in viscosity of a suspension due to the presence of spherical colloidal particles.

$$
\eta=\eta_{0}(1+2.5 \varphi)
$$

where, $\eta$ is the viscosity of suspension, $\eta_{0}$ is the viscosity of the incompressible fluid and $\varphi$ is the volume fraction of the spherical particles.

Guth and Gold [14] adapted the viscosity law to predict the small strain modulus of an elastomer filled with rigid spherical particles and they included an additional term to account for the interaction of fillers at larger filler volume fractions. They proposed that the increase in the modulus due to the incorporation of spherical fillers was given by,

$$
E=E_{0}\left(1+2.5 \varphi+14.1 \varphi^{2}\right)
$$

Where, $E$ is the modulus of the filled rubber, $E_{0}$ is the modulus of the matrix and $\varphi$ is the filler volume fraction. This relation assumes that the filler particles are spherical, well dispersed throughout the matrix, and that each is fully coated with rubber. These assumptions do not represent a realistic rubber-filler composite, as the fillers are not typically just spherical in shape and they tend to agglomerate. As a result, Guth [15] derived another equation where the filler particles are considered to have a representative average aspect ratio $f$ defined as the ratio of its longest length to its perpendicular breadth.

$$
E=E_{0}\left(1+2.5 f \varphi+14.1 f^{2} \varphi^{2}\right)
$$

An attempt was made to fit the experimental data using Eq. (3) and Eq.(4) with $\mathrm{E}_{0}=1.2 \mathrm{MPa} \quad(f=4)$ and $0.065 \mathrm{MPa}(f=12)$, which are the measured values for the elastic modulus for the unfilled NR and IIR samples respectively. A good fitting was obtained for low volume fraction loading of graphite up to 0.21 for NR and 0.25 for IIR which are going to be taken as the percolation thresholds for such system as will be assured by other models. However, the observed deviation from the linear behavior for higher volume fraction may be due to the limited applicability of the Guth's model for small volume fractions of the filler. Also, as the graphite loading increase, the filler filler and/or filler - matrix increases, which was not taken into account by Guth's model, and this may be the reason for the tremendous increase in the modulus with filler loading higher than the percolation threshold.

The value of the percolation threshold in filled elastomers can be determined through the analysis of Huber and Vilgis. This analysis which was proposed for 
carbon black $(\mathrm{CB})$ filled rubbers, predicts that the excess modulus of the filled elastomer $\left(E-E_{0} / E_{0}\right)$ changes linearly with the filler content below and above the percolation value with different slopes. The intersection point defines the percolation value [16].

In Figures (5) and (6), the initial modulus excess data are plotted as a function of the filler volume fraction in log-log scale. It is evident that, two regimes exist of both low and high filler volume fraction, each of which is described by a straight line with exponents 0.85 and 1.5 for NR and 0.98 and 2.64 for IIR respectively. The percolation threshold as being identified by the intersection points turns out to be 0.21 and 0.25 (corresponding to $40 \mathrm{phr}$ and $50 \mathrm{phr}$ ) for NR and IIR composites respectively, which are the same values obtained previously.

In order to account for the experimental data above the percolation threshold, the power law proposed for jammed systems by Trappe el al [17] could be used.

$$
E \propto\left(\varphi-\varphi_{c}\right)^{n}
$$

Or it may be rewritten in the form

$$
E=E_{c}+A\left(\varphi-\varphi_{c}\right)^{n}
$$

Where $E_{\mathrm{c}}$ is the elastic modulus of the composite at $\varphi_{c}, A$ and $\mathrm{n}$ are constants that depend on the filler volume fraction and the constituent of the composite. However, the value of $A$ may be taken as a measure of the filler interactions inside the composite. Fitting the experimental data using Eq.(6) gives us the values of the fitting parameters $A$ and $n$ which are tabulated in table (3).

\section{Membrane Samples}

It becomes obviously important to find how the preparation conditions and the final form of the composite may affect the properties of elastomeric composites. Therefore, in the present part, the effect of the membrane form and preparation conditions on the mechanical properties of NR as well as IIR loaded with graphite will be studied.

The stress-strain curves of the two groups of samples in the membrane state are shown in Figures (7) and (8). We can observe the same behavior for both composites as in the bulk state. That is the materials stiffness and strength increase as the graphite loading increase. The initial modulus behavior does not change appreciably than the case of bulk composites, but its value increases by about 18 times for NR than that of the bulk case for $100 \mathrm{phr}$ graphite loading. While for IIR matrix in the membrane form is slightly lower than that for bulk composite with 100 phr graphite loading. 
The experimental data in Figures (9) and (10) represent the dependence of the initial modulus of composites on the graphite volume fraction for both NR and IIR matrices respectively. The experimental data were fitted using Guth' Eq. (4) using $E_{0}=0.469 \mathrm{Mpa}$ for NR and $E_{0}=0.326 \mathrm{MPa}$ for IIR membrane matrices. A good fitting was obtained using $f=6$ for NR up to $\varphi=0.25$ for NR, while the fitting was good for IIR using $f=4$ for all volume fractions of graphite.

On applying Huber-Vilgis model for the NR in membrane state as shown in Fig. (11), one can determine the value of the percolation as was done for the Bulk composites. The percolation threshold obtained is 0.168 which is small value compared with the value obtained in the bulk state.

Finally, on applying the power law proposed for jammed systems, for NR membrane which fitted well the experimental data above the percolation threshold. The fitting parameters $A$ and $n$ for NR in membrane form are listed also in Table (3).

\section{Conclusion}

Composite samples were prepared in two final forms namely, the bulk and the membrane forms using graphite as reinforcing filler. Membrane form has a lower percolation threshold and a higher initial modulus than the bulk form. Huber-Vilgis model was successful in determining the percolation threshold of $\mathrm{s}$, while Guth' model failed in fitting the experimental data.

\section{References}

1. X. Yang, C. Grosjean, Y. Tai. J Microelectromech Syst 8, 393(1999).

2. S. M. Ha, W. Yuan, Q. Pei, R. Pelrine, S. Stanford. Adv Mater 18, 887 (2006).

3. L. Liu, Y. L. Lu, L. He, W. Zhang, C. Yang, Y. D. Liu, Adv Funct Mater 15, 309 (2005)

4. G. R. Hamed. Rubber Chem Technol 73, 524 (2000)

5. L. Q. Zhang, D. M. Jia. In: Symposium of international rubber conference, Beijing, China, September 21-25, vol. A, 46 (2004).

6. F. He, J. Fan, S. Lau, Poly. Testing 27, 970-964 (2008)

7. S. H. Song, H. K. Jeong, Y. G. Kang, and C.T. Cho, Korean J. Chem. Eng. 27(4), 1296-1300 (2010)

8. M. Sarikanat , K. Sever , E. Erbay , F. Güner, I. Tavman, A. Turgut , Y. Seki , I. Özdemir, Archives of Materials Sci. \& Eng. 50(2), 120-124 (2011)

9. L. L. Wang, L. Q. Zhang, M. Tian. Materials and Design 39, 450-457 (2012)

10. M. A. Raza, A.V. K. Westwood, A. P. Brown, C. Stirling, Composites Sci. and Tech. 72, 467-475 (2012) 
11. M. Amin, G. M. Nasr, G. H. Ramzy, E. Omar. J. Mater. Environ. Sci. 4 (5), 709-714 (2013)

12. A. Einstein, Ann. d. Physik 19, 289 (1906)

13. A. Einstein, Ann. d. Physik 34, 591 (1911)

14. E. Guth, and O. Gold, Phys. Rev. 53, 322 (1938).

15. E. Guth, J. Appl. Phys. 16, 20 (1945).

16. G. Huber, and T.A. Vilgis, Kaut. Gummi. Kunstst. 52(2),102 (1999)

17. V. Trappe, V. Prasad, and L. Cipelletti, P.N. Segre, and D.A. Weitz, Nature 411, 772 (2001) 


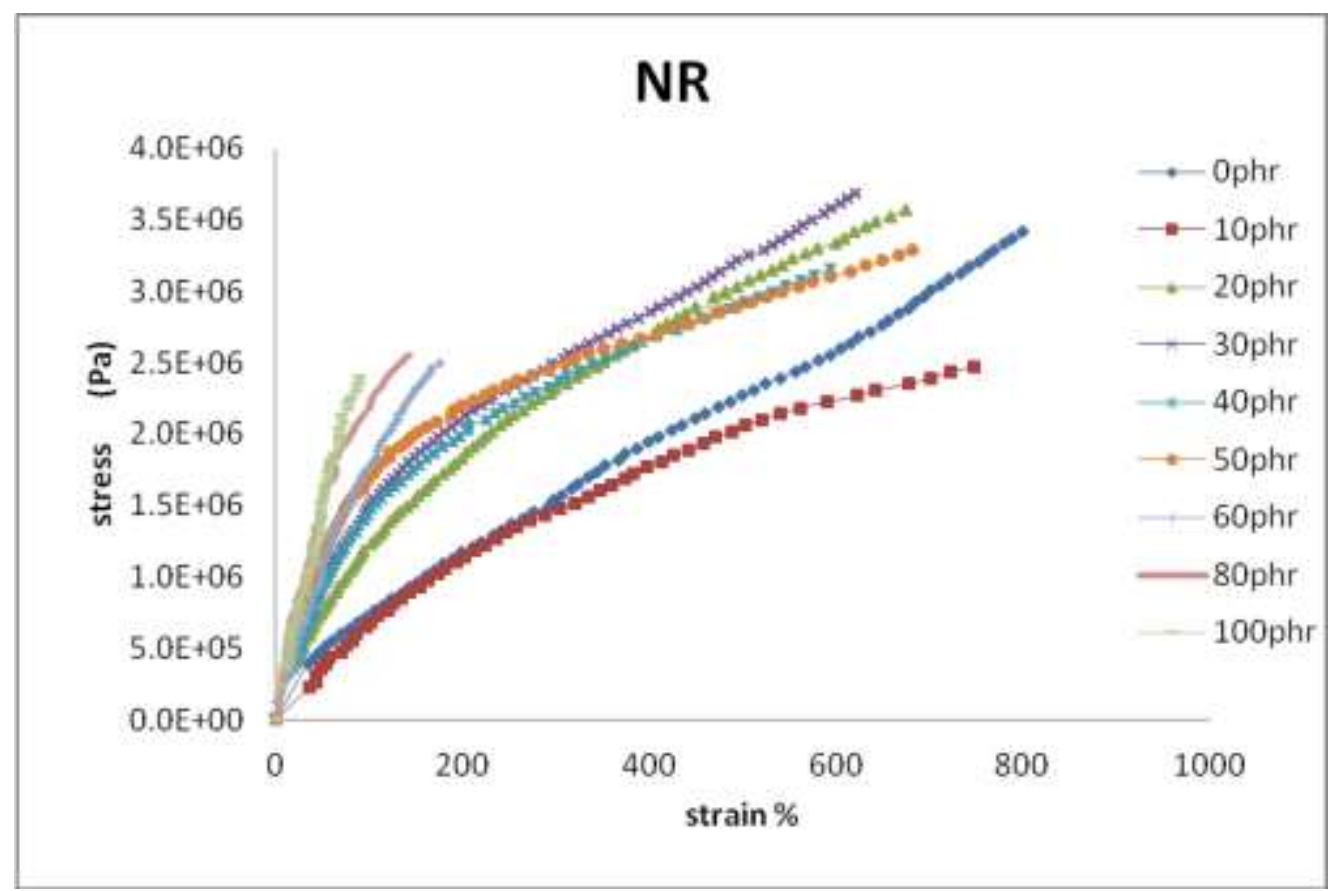

Figure (1) Stress-strain curves obtained in tensile tests performed at room temperature for different volume fractions of NR/graphite composites. 


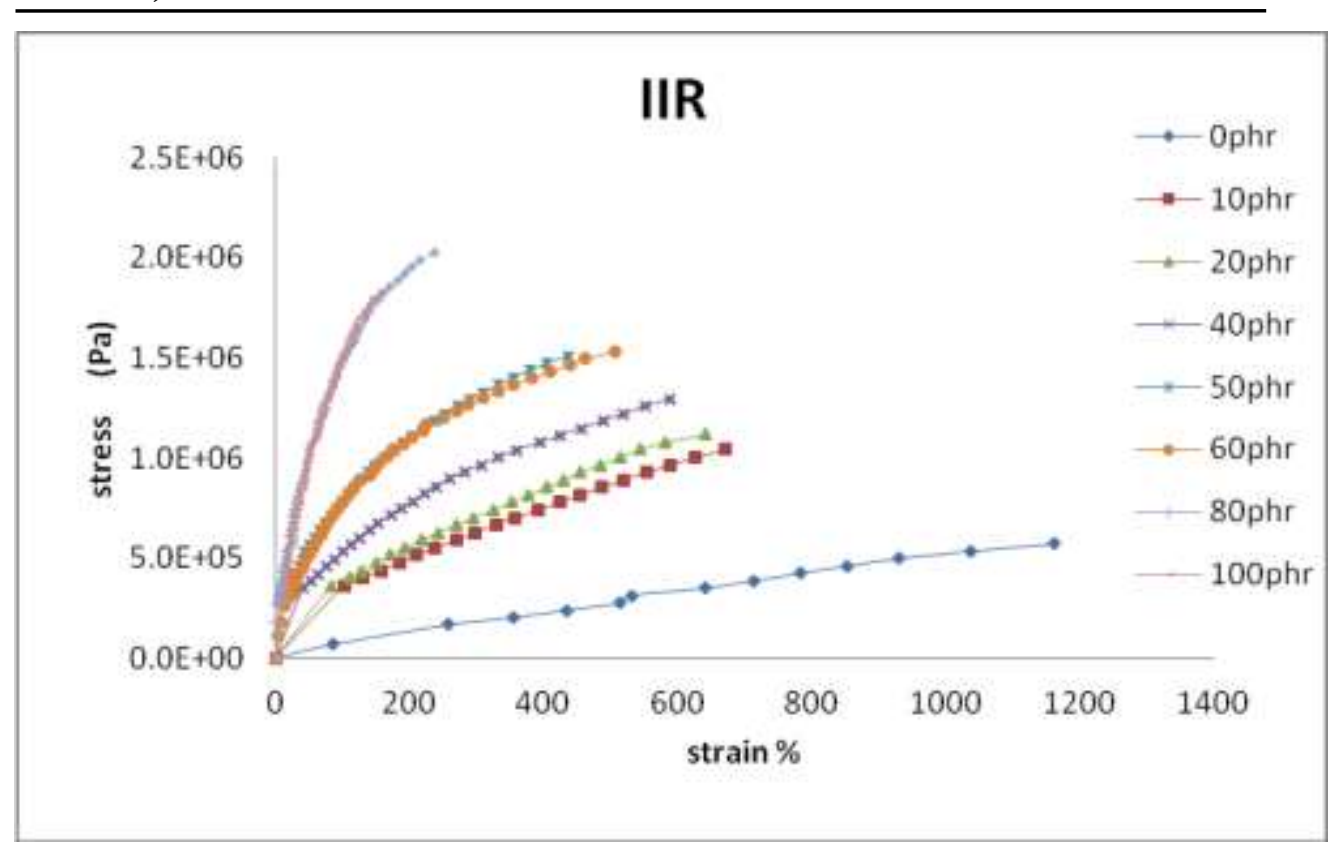

Figure (2) Stress-strain curves obtained in tensile tests performed at room temperature for different volume fractions of IIR/graphite composites. 
NR

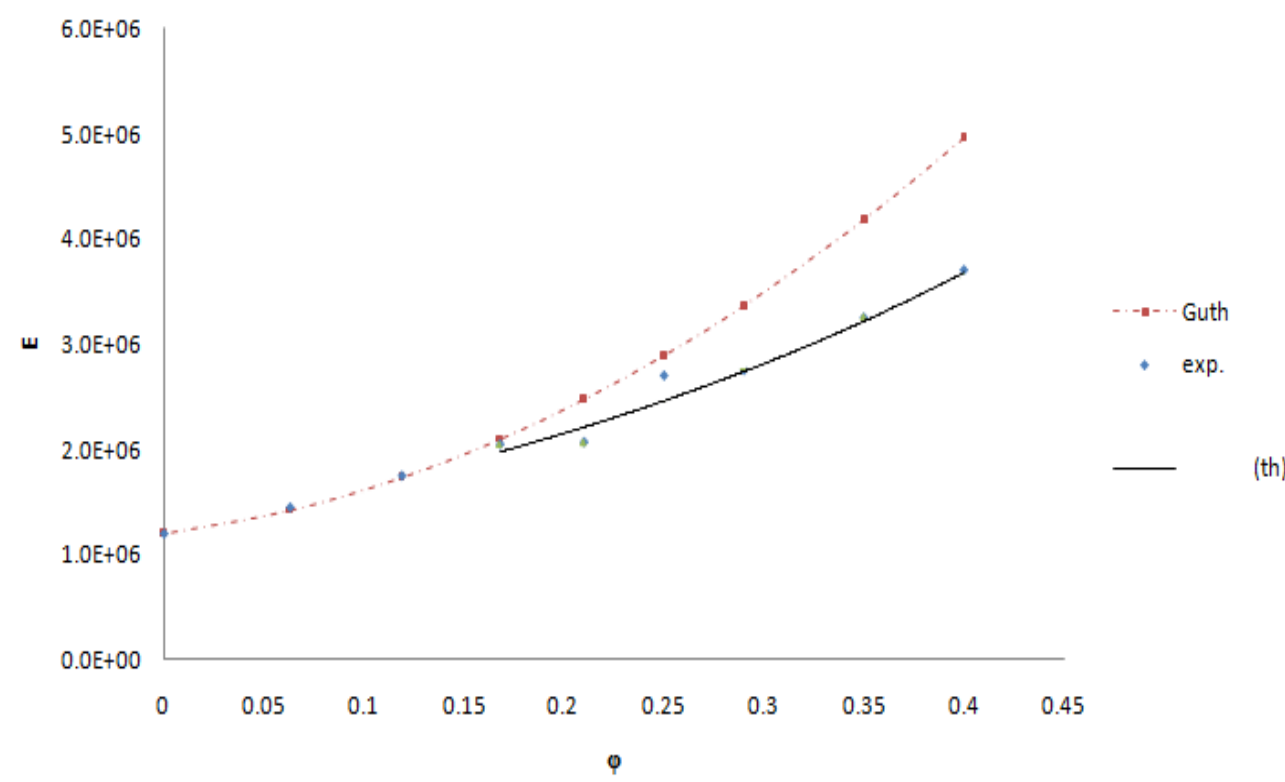

Figure (3) Initial modulus, evaluated from the slope of the curves of Fig.(1), as a function of the true graphite volume fraction. Data were taken at room temperature. 


\section{IIR}

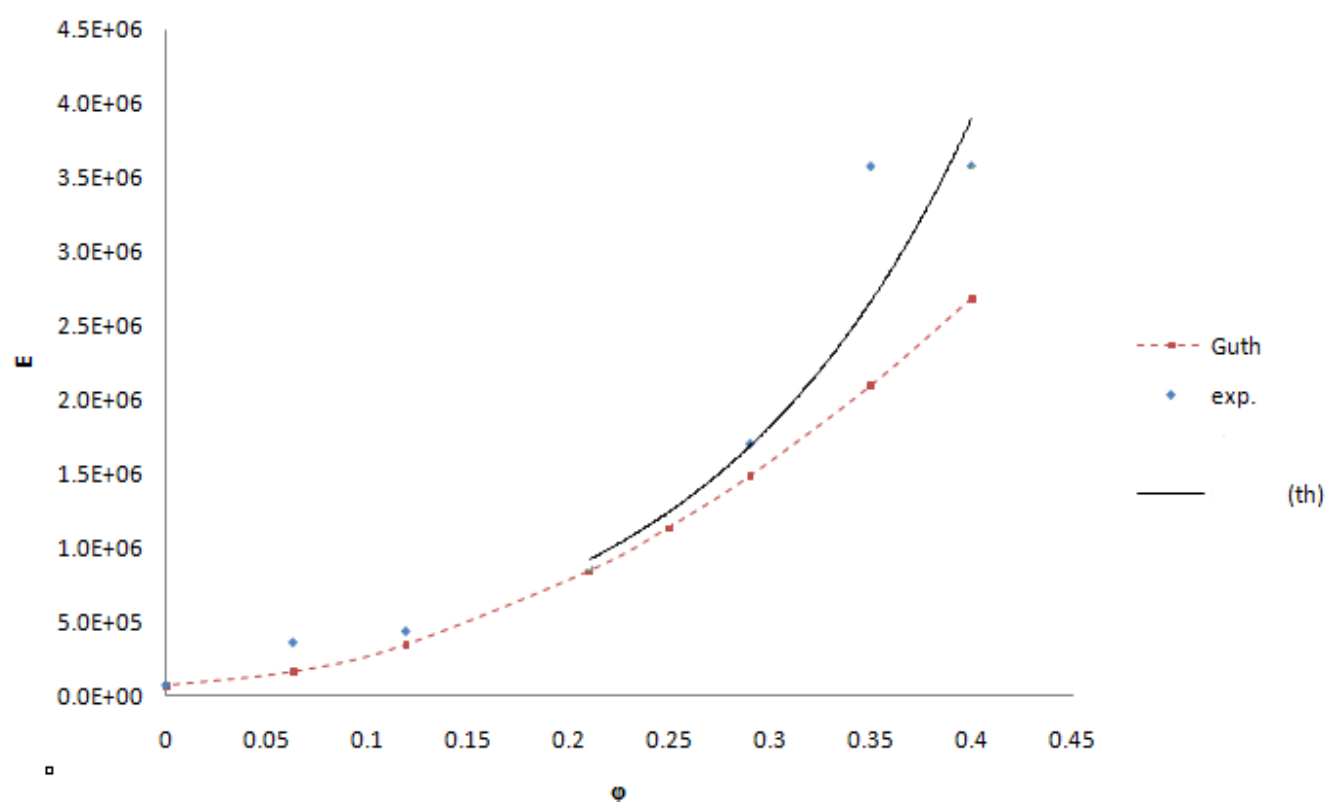

Figure (4) Initial modulus, evaluated from the slope of the curves of Fig.(2), as a function of the true graphite volume fraction. Data were taken at room temperature. 




Figure (5) Log-log plot of the excess modulus as a function of the true graphite loading for NR (Huber-Vilgis plot). 


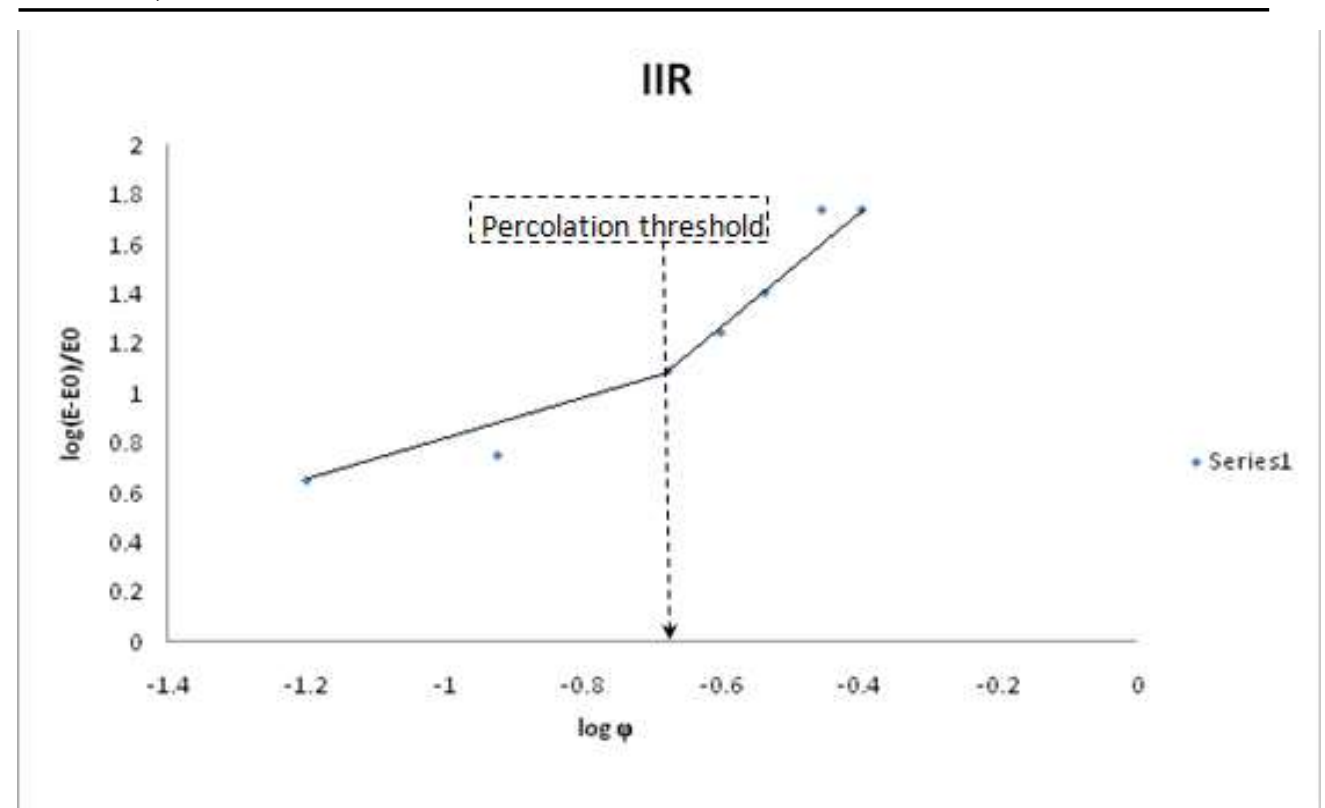

Figure (6) Log-log plot of the excess modulus as a function of the true graphite loading for IIR (Huber-Vilgis plot). 


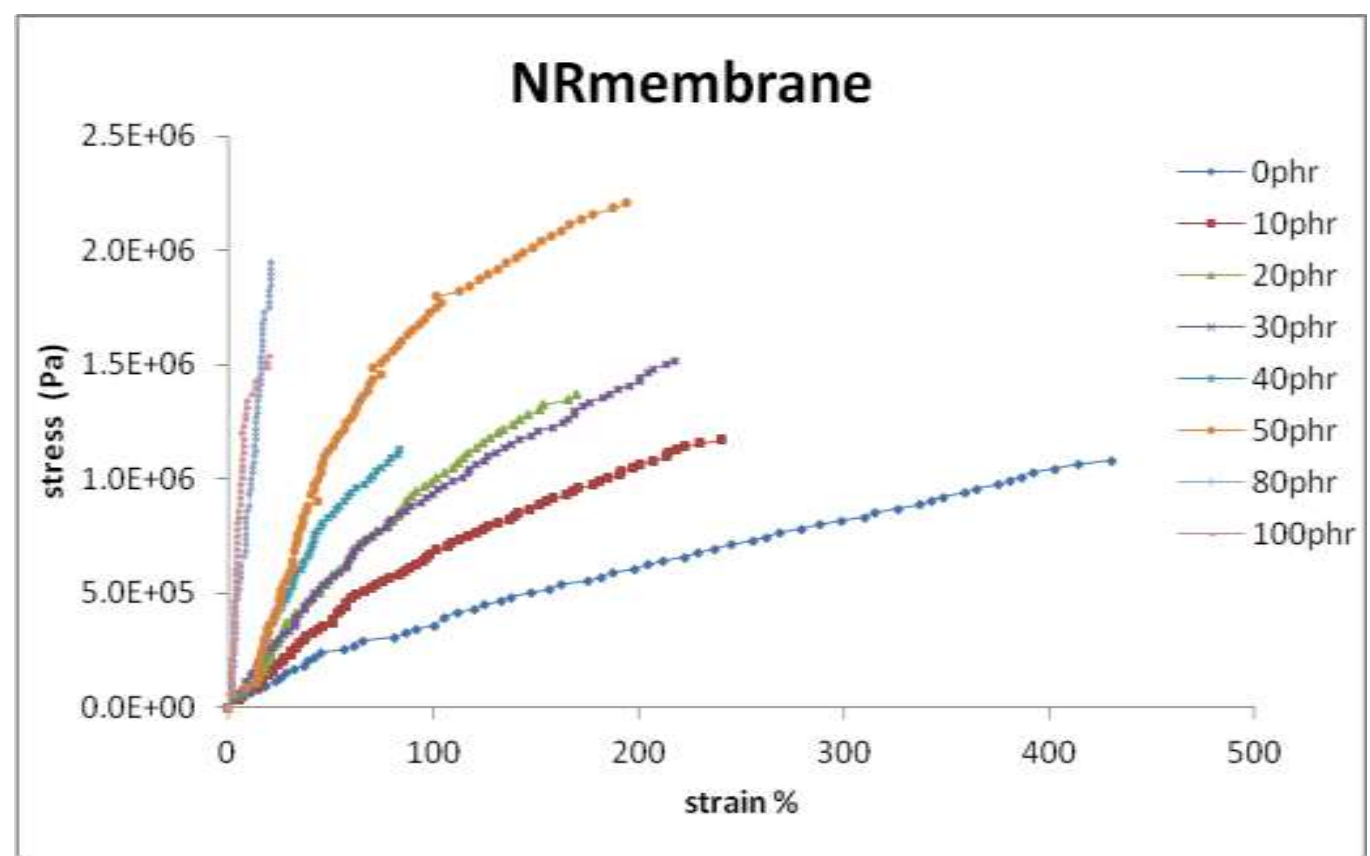

Figure (7) Stress-strain curves obtained in tensile tests performed at room temperature for different volume fractions of NR membrane/graphite composites. 


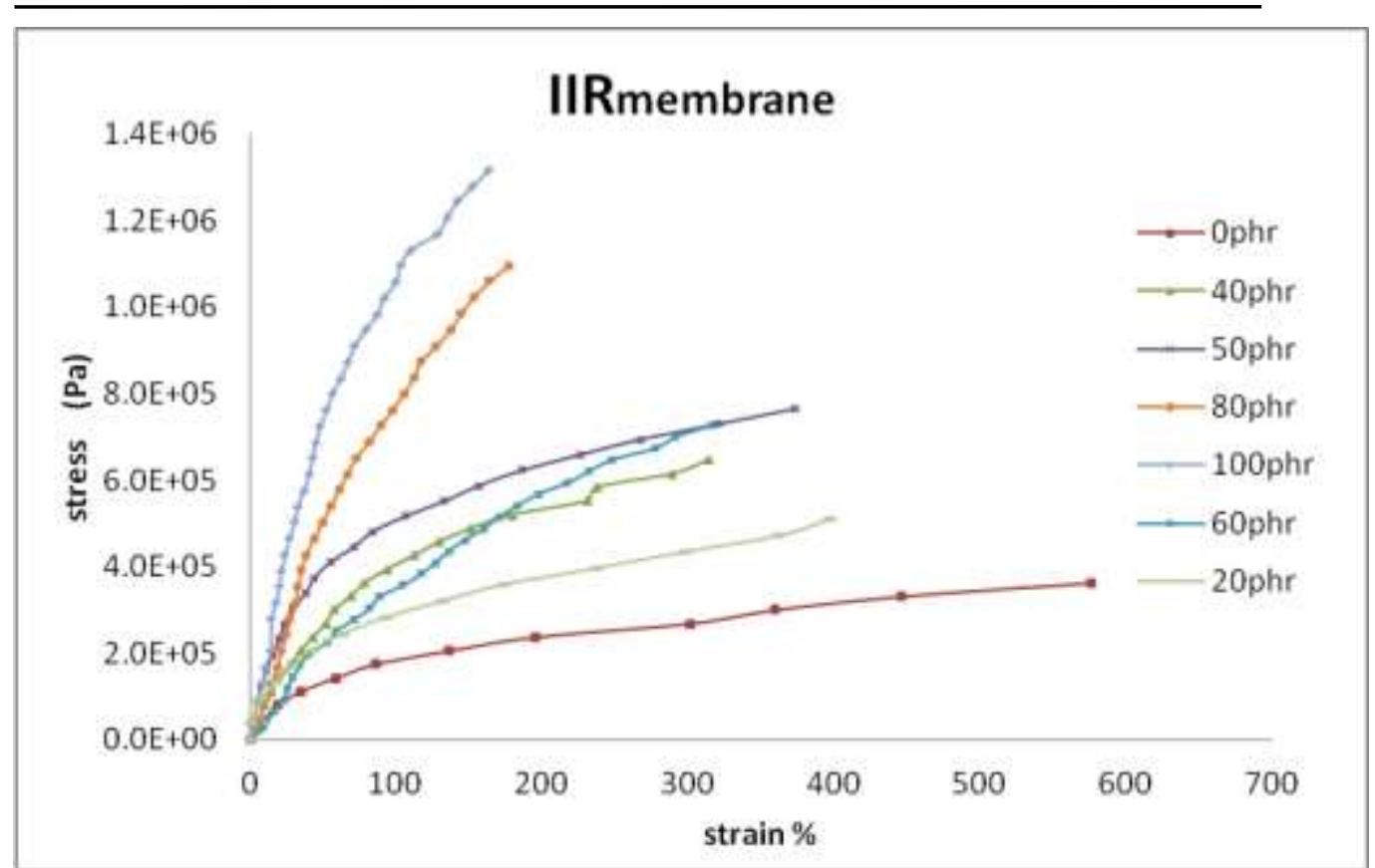

Figure (8) Stress-strain curves obtained in tensile tests performed at room temperature for different volume fractions of IIR membrane/graphite composites. 


\section{NRmembrane}



Figure (9) Initial modulus, evaluated from the slope of the curves of Fig.(7), as a function of the true graphite volume fraction for NR. Data were taken at room temperature. 


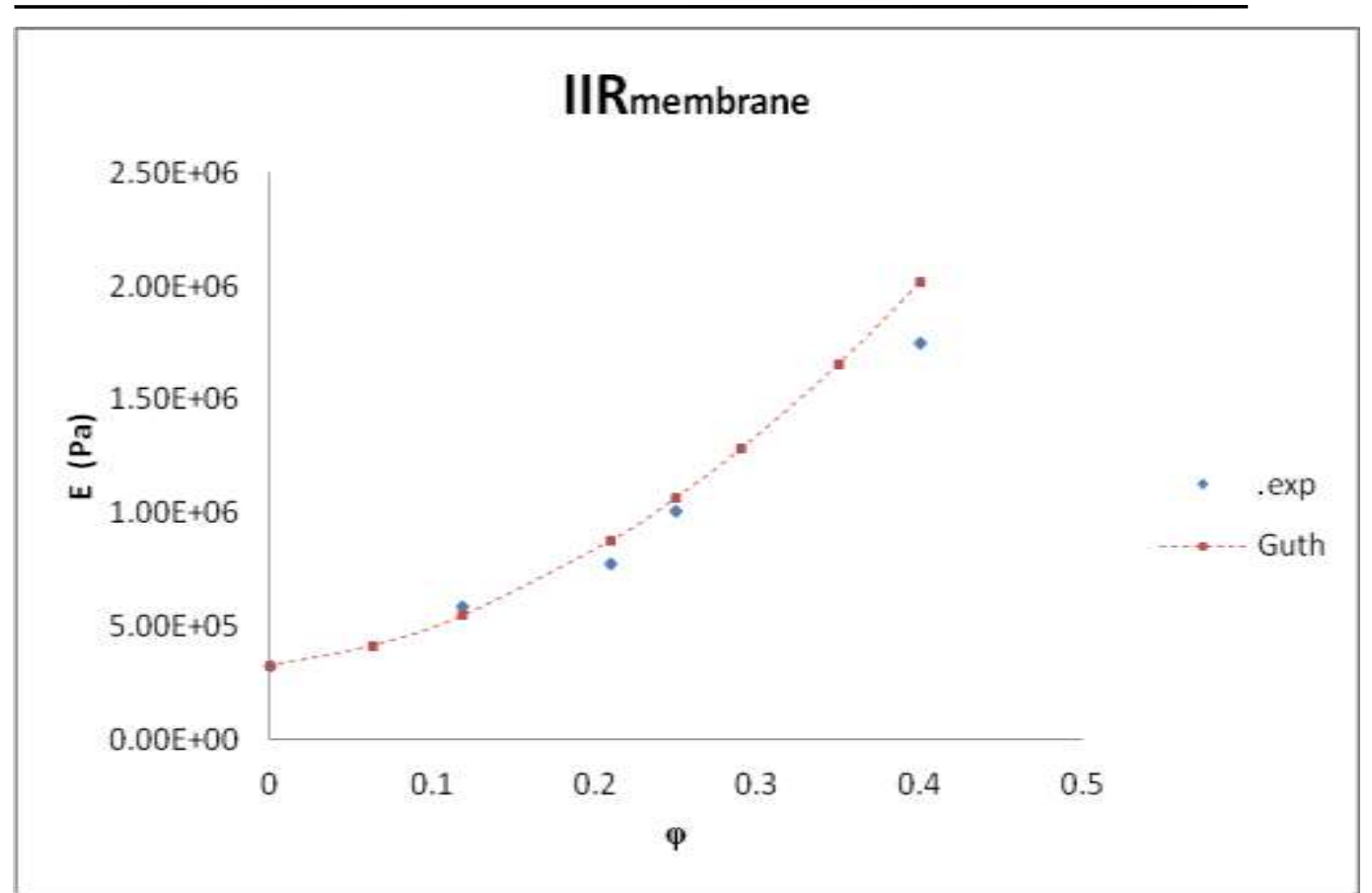

Figure (10) Initial modulus, evaluated from the slope of the curves of Fig.(8), as a function of the true graphite volume fraction for IIR. Data were taken at room temperature. 


\section{NRmembrane}



Figure (11) Log-log plot of the excess modulus as a function of the true graphite loading for membrane NR (Huber-Vilgis plot).

Table 1

Formulation of the rubber compounds

\begin{tabular}{|c|c|c|}
\hline Quantity $\left(\mathbf{p h r}^{\mathbf{a}}\right)$ & \multicolumn{2}{|c|}{ Ingredients } \\
\hline 100 & NR & Rubber \\
\hline $0,10,20,30,40,50,60,80,100$ & Graphite & Filler \\
\hline 10 & Processing oil & Plasticizer \\
\hline
\end{tabular}




\begin{tabular}{|c|c|c|}
\hline 1.5 & Stearic acid & \multirow{2}{*}{ Activators } \\
\cline { 1 - 2 } 5 & Zinc oxide & Accelerator \\
\hline 1.5 & $\mathrm{MBTS}^{\mathrm{b}}$ & Age resisters \\
\hline 1 & $\mathrm{PBN}^{\mathrm{c}}$ & Vulcanizing Agents \\
\hline
\end{tabular}

${ }^{a}$ Parts per hundred parts by weight of rubber

Table (2)

Composition of IIR composite

\begin{tabular}{|c|c|}
\hline Quantity $\left(\mathbf{p h r}^{\mathbf{a}}\right)$ & Ingredients \\
\hline 100 & IIR \\
\hline $0,10,20,40,50,60,80,100$ & Graphite \\
\hline 10 & Processing oil \\
\hline 1.5 & Stearic acid \\
\hline 5 & Zinc oxide \\
\hline 1.5 & MBTS $^{\mathrm{b}}$ \\
\hline 1 & PBN $^{\mathrm{c}}$ \\
\hline 2 & Sulfur \\
\hline
\end{tabular}

a Parts per hundred parts by weight of rubber

b Dibenzthiazyle disulphide

c Pheny1- $\beta$-naphthylamine 
Table (3)

The fitting parameters $A$ and $n$ of Equation (6) for NR and IIR groups.

\begin{tabular}{|c|c|c|c|}
\hline \multicolumn{3}{|c|}{ Group of samples } & The fitting parameters \\
\hline NR membrane & IIR & NR & \\
\hline 0.8 & 0.9 & 0.9 & $\boldsymbol{n}$ \\
\hline 86 & 13 & 7 & $\boldsymbol{A}(\mathbf{M P a})$ \\
\hline
\end{tabular}

